\title{
A Conjecture on a Matrix Group With Two Generators
}

\section{Morris Newman}

\author{
Institute for Basic Standards, National Bureau of Standards, Washington, D.C. 20234
}

(January 3, 1973)

Let $\zeta$ be a primitive $q$ th root of unity. It is conjectured that the group generated by

$$
A=\left(\begin{array}{ll}
1 & \zeta \\
0 & 1
\end{array}\right) \quad \text { and } \quad B=\left(\begin{array}{ll}
1 & 0 \\
\zeta & 1
\end{array}\right)
$$

is never free. The conjecture is proved when $q$ is an even prime power, or an odd prime power having 2 as a primitive root.

Key words: Free groups; matrix groups; roots of unity.

Let $G=\{A, B\}$ be the group generated by the matrices

$$
A=\left(\begin{array}{ll}
1 & \zeta \\
0 & 1
\end{array}\right), \quad B=A^{T}=\left(\begin{array}{ll}
1 & 0 \\
\zeta & 1
\end{array}\right)
$$

where $\zeta$ is an arbitrary complex number. It has been known for some time that $G$ is free when $|\zeta| \geqslant 2$ (see [1], [3]); and it is also true that $G$ is free if $\zeta$ is transcendental, or if $\zeta$ is algebraic and has a conjugate which is greater than or equal to 2 in absolute value. (See the references at the end of this note for further results of this kind.) The only values in question therefore are those algebraic $\zeta$ all of whose conjugates are less than 2 in absolute value. This remark prompts the conjecture that if $\zeta$ is a root of unity, then $G$ is not free. Although we do not have a proof of this, the following result provides evidence that it is correct:

THEOREM: Suppose that $\zeta$ is a primitive q th root of unity, where q is a prime power. Then G is not free when $\mathrm{q}$ is even, and also when $\mathrm{q}$ is odd and 2 is a primitive root of $\mathrm{q}$.

Proof: We define a sequence of elements of $G$ as follows:

$$
K_{1}=B=\left(\begin{array}{ll}
1 & 0 \\
\zeta & 1
\end{array}\right), \quad K_{m+1}=K_{m} A^{-1} K_{m}^{-1}=K_{m}\left(\begin{array}{rr}
1 & -\zeta \\
0 & 1
\end{array}\right) K_{m}^{-1}, \quad m \geqslant 1 .
$$

We note first that as a formal word in $A$ and $B$ no cancellation occurs, and that $K_{m}$ is of length $2^{m}-1$, beginning and ending with $B$. Next, set

$$
K_{m}=\left(\begin{array}{ll}
a_{m} & b_{m} \\
c_{m} & d_{m}
\end{array}\right), \quad m \geqslant 1 .
$$

Then (1) implies readily that for $m \geqslant 1$,

$$
\begin{array}{ll}
a_{m+1}=1+\zeta a_{m} c_{m}, & b_{m+1}=-\zeta a_{m}^{2} \\
c_{m+1}=\zeta c_{m}^{2}, & d_{m+1}=1-\zeta a_{m} c_{m},
\end{array}
$$

AMS Subject Classification: 20F05; $20 \mathrm{H} 10$. 
from which we deduce that for $m \geqslant 1$,

(2)

$$
a_{m}=\sum_{k=0}^{m-1} \zeta^{2^{m}-2^{k+1}}, \quad b_{m}=-\zeta a_{m-1}^{2},
$$

$$
c_{m}=\zeta^{2 m-1}, \quad d_{m}=2-a_{m},
$$

where $a_{0}$ is understood to be 0 .

Suppose first that $q=2^{r}$, so that $\zeta^{2 r}=1, \zeta^{2^{r}-1}=-1$. Choose $m=r-1$. Then $\operatorname{tr}\left(A K_{m}\right)=a_{m}+$ $\zeta c_{m}+d_{m}=2+\zeta^{2 m}=1$, and so $\left(A K_{m}\right)^{6}=\mathrm{I}$. This is a genuine relation, so that $G$ is not free in this case.

Next suppose that $q=p^{r}$, where $p$ is an odd prime and 2 is a primitive root of $q$. Then the numbers $2^{k}, 0 \leqslant k \leqslant \varphi(q)-1$, form a reduced set of residues modulo $q$. Choose $m=1+\varphi(q)$. Then it follows easily that

$$
a_{m}=\sum_{k=0}^{\varphi(q)} \zeta^{2^{m}-2^{k+1}}=1+\zeta^{2} \mu(q),
$$

where $\mu(q)$ is the Möbius function, since the numbers

$$
\zeta^{-2^{k+1}}, \quad 0 \leqslant k \leqslant \varphi(q)-1
$$

are the $\varphi(q)$ primitive $q$ th roots of unity, and the sum of the primitive $q$ th roots of unity is $\mu(q)$. There are now two subcases to consider.

I. $r>1$. Then $\mu(q)=0, \quad$ and $a_{m}=1$.

It follows from (2) that $K_{m}=\left(\begin{array}{ll}1 & 0 \\ \zeta & 1\end{array}\right)=B$, which is a genuine relation.

II. $r=1$. Then $\mu(q)=-1, \quad a_{m}=1-\zeta^{2}$,

$$
\begin{aligned}
K_{m} & =\left(\begin{array}{ll}
1-\zeta^{2} & -\zeta^{3} \\
\zeta & 1+\zeta^{2}
\end{array}\right) . \text { Thus } \\
A K_{m} & =\left(\begin{array}{ll}
1 & \zeta \\
0 & 1
\end{array}\right) K_{m}=\left(\begin{array}{cc}
1 & \zeta \\
\zeta & 1+\zeta^{2}
\end{array}\right)=B A, \\
K_{m} & =A^{-1} B A . \text { Again, this is a genuine relation. }
\end{aligned}
$$

This completes the proof.

\section{References}

[1] Brenner, J. L., Quelques groupes libres de matrices, C. R. Acad. Sci. Paris 241, 1689-1691 (1955).

[2] Chang, B., Jennings, S. A., and Ree, R., On certain pairs of matrices which generate free groups. Canad. J. Math. 10, 279-284 (1958).

[3] Lyndon, R. C., and Ullman, J. L., Pairs of real 2-by-2 matrices that generate free products, Mich. Math. J. 15, 161-166 (1968).

[4] Newman, M., Pairs of matrices generating discrete free groups and free products, Michigan Math. J. 15, 155-160 (1968).

(Paper 78B2-400) 